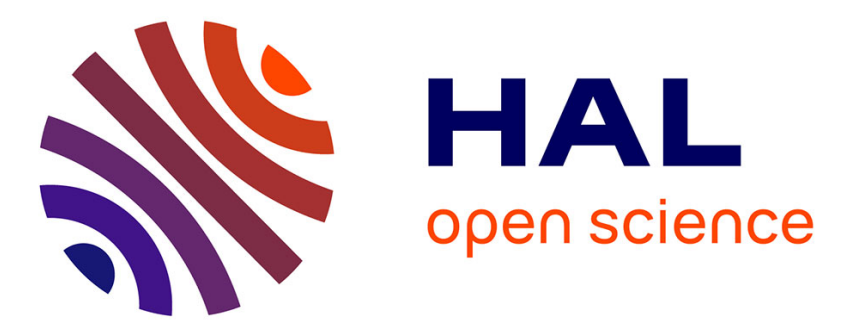

\title{
SYNTHĖSE NUMÉRIQUE DE SOURCES INDUSTRIELLES POUR LE TEST DES MÉTHODES D'HOLOGRAPHIE ACOUSTIQUE
}

\author{
J. Li, A. Garcia, C. Carles, J. Pascal
}

\section{- To cite this version:}

J. Li, A. Garcia, C. Carles, J. Pascal. SYNTHÈSE NUMÉRIQUE DE SOURCES INDUSTRIELLES POUR LE TEST DES MÉTHODES D'HOLOGRAPHIE ACOUSTIQUE. Journal de Physique IV Proceedings, 1992, 02 (C1), pp.C1-753-C1-756. 10.1051/jp4:19921164 . jpa-00251124

HAL Id: jpa-00251124

https://hal.science/jpa-00251124

Submitted on 1 Jan 1992

HAL is a multi-disciplinary open access archive for the deposit and dissemination of scientific research documents, whether they are published or not. The documents may come from teaching and research institutions in France or abroad, or from public or private research centers.
L'archive ouverte pluridisciplinaire HAL, est destinée au dépôt et à la diffusion de documents scientifiques de niveau recherche, publiés ou non, émanant des établissements d'enseignement et de recherche français ou étrangers, des laboratoires publics ou privés. 


\title{
SYNTHÈSE NUMÉRIQUE DE SOURCES INDUSTRIELLES POUR LE TEST DES METHODES D'HOLOGRAPHIE ACOUSTIQUE
}

\author{
J.F. LI, A. GARCIA, C. CARLES et J.C. PASCAL* \\ Laboratoire d'Acoustique, Conservatoire National des Arts et Métiers (CNAM), 292 rue Saint-Martin, \\ F-75141 Paris cedex 03, France \\ ${ }^{*}$ Centre Technique des Industries Mécaniques (CETIM), 52 avenue Félix-Louat, F-60300 Senlis, \\ France
}

\begin{abstract}
SUMMARY - A numerical simulation model is presented for synthesizing the sound field by point sources (monopoles, dipoles etc) located in the space. Results are given in order to show the influence of the source coherence matrix to the sound radiation. The relationships between the source reference (in which the acoustic sources are defined) and the measuring reference (in which the measurements are made) are established in order to meet the need of the pratical traitement of acoustical measurement. By means of these transformations, the sound fields (the acoustic intensity, the density of potential energy and kinetic energy) can be easily illustrated on the rectangular plan or on the cylindrical surface, which could have any orientation and position with respect to the sound sources. From the results we have given several indications about the location and the characterization of the sound fields.
\end{abstract}

\section{Introduction}

Des modèles de sources ponctuelles (monopôles, dipôles et quadrupôles) sont souvent utilisés pour étudier les champs acoustiques [1,2]. La superposition du champ de plusieurs de ces sources élémentaires permet de simuler des situations complexes telles que celles qui sont produites par des sources réelles [3]. Pour notre part, nous avons développé des modèles numériques permettant le calcul du rayonnement acoustique dans l'espace produit par des sources ponctuelles monopolaires et dipolaires, en prenant en compte la notion de cohérence entre sources élémentaires. Ces modèles permettent d'étudier des rayonnements acoustiques dans le cas de situations harmoniques (champ totalement corrélé) et dans le cas de situations aléatoires (champ partiellement corrélé). Pour simuler la mesure d'une source réelle, on établit une transformation entre le repère de source et le repère où sont définis les points de mesure. Cette transformation permet de représenter le champ acoustique sur une surface (plan ou cylindre) dont la position et l'orientation sont quelconques. Ces modèles sont utilisés pour évaluer les performanees des algorithmes d'analyse basés sur l'intensité acoustique et la méthode d'holographie acoustique BAHIM [4].

\section{Modèle de source complexe}

On considère un modèle de source constitué par un ensemble de sources acoustiques élémentaires ponctuelles monopolaires et dipolaires. Pour chaque source élémentaire, on définit son amplitude $A_{i}$, sa phase $\beta_{i}$ (par rapport à une référence arbitraire) et sa position dans l'espace $\left(x_{i}, y_{i}, z_{i}\right)$. Ainsi, pour un monopôle $i$, sa pression acoustique est $p_{i}(x, y, z)=A_{i} \exp \left[-j\left(k R_{i}-\beta_{i}\right)\right] / R_{i}$, où $R_{i}=\sqrt{\left(x-x_{i}\right)^{2}+\left(y-y_{i}\right)^{2}+\left(z-z_{i}\right)^{2}}$. Pour un dipôle on définit en plus l'orientation $\mathbf{D}_{i}$ de son axe par les angles $\theta_{d_{i}}$ et $\phi_{d_{i}}$ (voir Figure 1). Sa pression s'exprime par

$$
p_{i}(x, y, z)=A_{i} \frac{e^{-j\left(k R_{i}-\beta_{i}\right)}}{R_{i}}\left(1-\frac{j}{k R_{i}}\right) \cos \xi_{i},
$$

$$
\cos \xi_{i}=\left[\left(x-x_{i}\right) \sin \theta_{d_{i}} \cos \phi_{d_{i}}+\left(y-y_{i}\right) \sin \theta_{d_{i}} \sin \phi_{d_{i}}+\left(z-z_{i}\right) \cos \theta_{d_{i}}\right] / R_{i}
$$


où $\xi_{i}$ est l'angle entre le vecteur $\mathbf{R}_{i}$ et le vecteur $\mathbf{D}_{i}$. Pour chaque type de source élémentaire, on a obtenu une expression analytique de la vitesse particulaire $u_{i}(x, y, z)=(-1 / j \rho c k) \nabla p_{i}(x, y, z)$. Pour le dipôle, une formule générale établie à partir des équations (1a) et (1b) incluant son orientation et sa position dans l'espace, permet de simplifier les calculs [5].
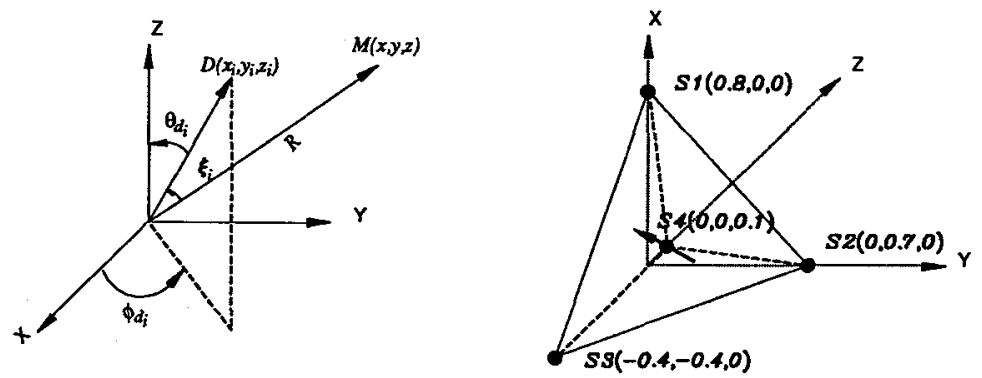

Figure 1 - Orientation des dipôles.

Figure 2 - Disposition des sources élémentaires (cf fig. 3)

Pour synthétiser des champs acoustiques semblables à ceux qui sont rayonnés par des sources industrielles, c'est à dire à spectre étendu, il est nécessaire de considérer les relations de cohérence entre les sources sous la forme d'une matrice de cohérence $\left[\gamma_{i j}^{2}\right]$. Les champs d'intensité acoustique active, d'intensité réactive et de densité d'énergie potentielle (ou pression quadratique) s'expriment respectivement sous les formes

$$
\mathbf{I}=\frac{1}{2} \operatorname{Re}\left\{\sum_{i=1}^{M} \sum_{j=1}^{M} \gamma_{i j} p_{i} \mathbf{u}_{j}^{*}\right\}, \quad \mathbf{J}=\frac{1}{2} \operatorname{Im}\left\{\sum_{i=1}^{M} \sum_{j=1}^{M} \gamma_{i j} p_{i} \mathbf{u}_{j}^{*}\right\}, \quad V=\frac{1}{4 \rho c^{2}} \sum_{i=1}^{M} \sum_{j=1}^{M} \gamma_{i j} p_{i} p_{j}^{*} .
$$

Dans le cas où les sources sont cohérentes, la matrice $\left[\gamma_{i j}^{2}\right]$ est unitaire et, dans ce cas seulement, on peut définir une pression et une vitesse particulaire complexe pour l'ensemble du champ telle que $p=\sum_{i=1}^{M} p_{i}$ et $\mathbf{u}=\sum_{i=1}^{M} \mathbf{u}_{i}$. Quand les sources sont indépendantes, $\left[\gamma_{i j}^{2}\right]$ est diagonale et les termes croisés n'existent pas. L'intensité active, par exemple, est la somme vectorielle des intensités de chaque source rayonnant séparément: $I=\sum_{i=1}^{M} \frac{1}{2} \operatorname{Re}\left\{p_{i} \mathbf{u}_{i}^{*}\right\}$. Dans le cas général, la matrice de cohérence sera définie pour l'ensemble des sources telle que $0 \leq \gamma_{i j}^{2} \leq 1$, pour $i \neq j$. La Figure 2 montre une source synthétique constituée par 3 monopôles et un dipôle $\left(\theta_{d}=\pi / 2, \phi_{d}=5 \pi / 6\right)$. Son champ rayonné à la fréquence de $500 \mathrm{~Hz}$ (intensité active et pression quadratique) est représenté sur la Figure 3 pour différentes valeurs de la matrice de cohérence.

\section{Transformation de repère}

Pour faciliter l'utilisation du logiciel, les points de l'espace où est calculé le champ acoustique sont définis dans un repère de mesure qui peut se déplacer par rapport au repère où sont définies les sources. Concrètement, si pour tester un algorithme d'holographie acoustique on veut obtenir une représentation du champ sur une antenne plane, il est facile de définir un maillage sur le plan $(x, y)$ d'un repère propre (repère de mesure) dans lequel seront ensuite représentées les données et qui est déplacé autour des sources par un nombre réduit de mouvements: translation $\left(x_{r_{0}}, y_{r_{0}}, z_{r_{0}}\right)$, rotation autour d'un axe vertical $\alpha_{1}$ et rotation autour d'un axe horizontal $\alpha_{2}$. Les coordonnées des points $\left(x_{m}, y_{m}, z_{m}\right)$ sont ensuite converties dans le repère des sources à l'aide d'une matrice de passage [5]

$$
\left[\begin{array}{l}
x_{r} \\
y_{r} \\
z_{r}
\end{array}\right]=M_{p} \times\left[\begin{array}{l}
x_{m} \\
y_{m} \\
z_{m}
\end{array}\right]+\left[\begin{array}{l}
x_{r_{0}} \\
y_{r_{0}} \\
z_{r_{0}}
\end{array}\right], \quad M_{p}=\left[\begin{array}{ccc}
-\sin \alpha_{1} & -\sin \alpha_{2} \cos \alpha_{1} & \cos \alpha_{1} \cos \alpha_{2} \\
\cos \alpha_{1} & -\sin \alpha_{2} \sin \alpha_{1} & \cos \alpha_{2} \sin \alpha_{1} \\
0 & \cos \alpha_{2} & \sin \alpha_{2}
\end{array}\right] .
$$

Une matrice de passage a également été définie pour des surfaces cylindriques. Ce sont les coordonnées des points de mesure dans le repère des sources qui sont utilisées pour le calcul des champs énergétiques. Pour 

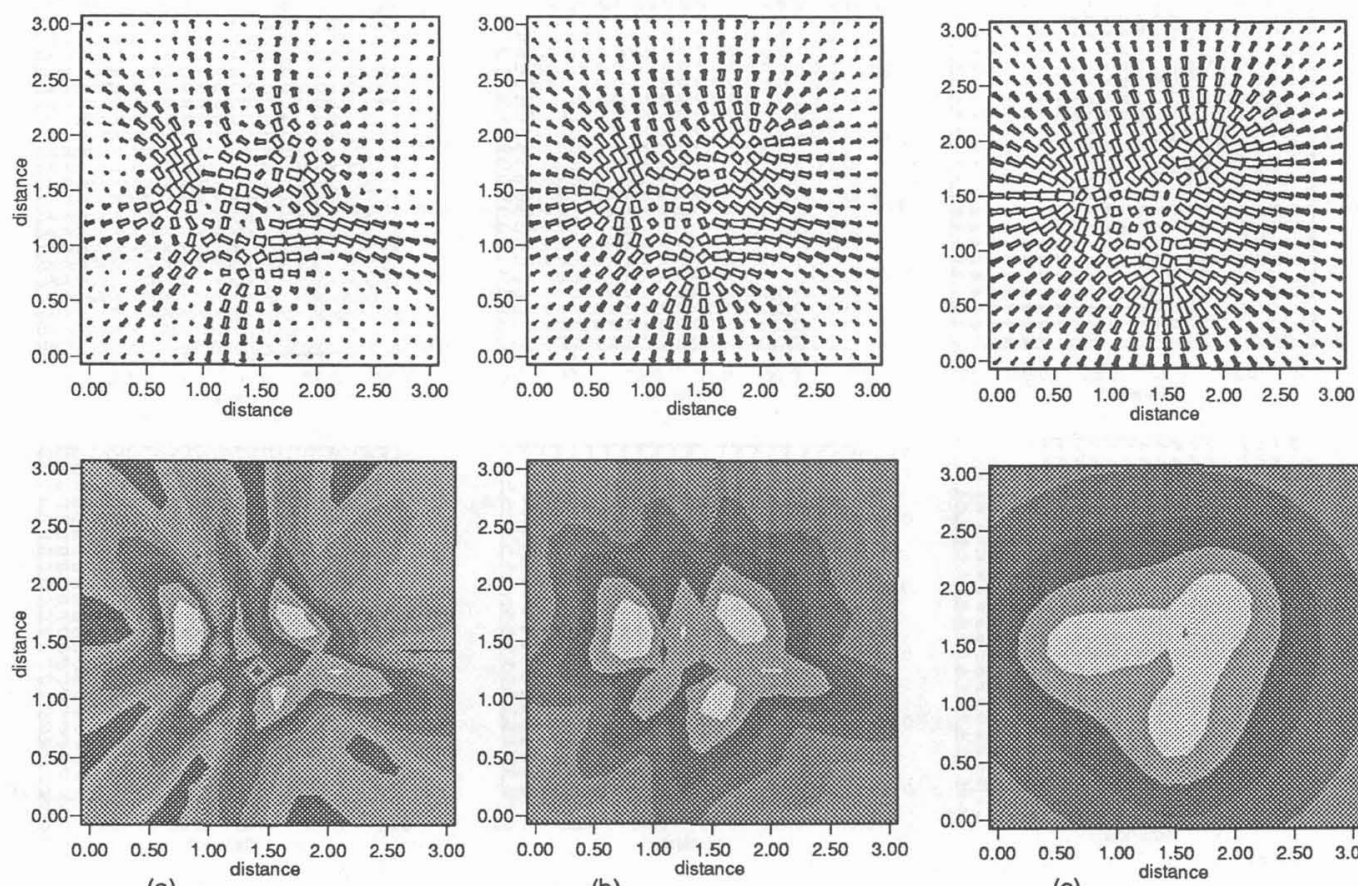

(a)

(b)

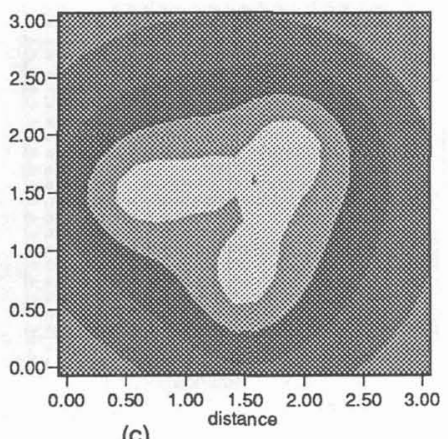

(c)

Figure 3 - Intensité acoustique (haut) et densité d'énergie potentielle (bas) sur un plan situé à $0.3 \mathrm{~m}$ dans la direction $z$ du centre de la source synthétique de la Figure 2 ; (a) $\gamma_{i j}^{2}=1$, (b) $\gamma_{i j}^{2}=0.5$, (c) $\gamma_{i j}^{2}=0$.

pouvoir représenter les quantités vertorielles (intensités et vitesse) dans le repère de mesure, il est encore nécessaire de leur appliquer la transformation inverse $\mathbf{V}_{m}=M_{p}^{-1} \mathbf{V}_{r}$, où les vecteurs $V_{r}$ et $V_{m}$ peuvent représenter des vitesses particulaires, des intensités active ou réactive, respectivement dans le repère des sources et dans le repère de mesure. L'utilisation des transformations de repère pour explorer le champ des sources est illustrée par la Figure 4. Le rayonnement d'un dipôle situé en $(0,0,0)$ et orienté dans la direction $x$ est calculé par les équations (1) et (2) et projeté sur des plans d'observation disposés diversement dans l'espace en appliquant les équations (3). On remarque sur ces figures que l'origine apparente des champs d'intensité active et des champs d'intensité réactive semble différente. Cette particularité, surprenante au premier abord, peut s'expliquer en considérant les relations suivantes:

$$
\mathbf{I}=-2 \frac{c}{k} V \nabla \varphi, \quad \mathbf{J}=-\frac{c}{k} \nabla V .
$$

La direction des vecteurs des intensités active et réactive est déterminée par le gradient de deux quantités scalaires très différentes : la phase du champ de pression et la densité d'énergie potentielle (ou pression quadratique). La trace de ces grandeurs scalaires sur un plan qui ne contient pas la source conduira généralement aux résultats que nous venons de constater, pour toutes les sources présentant une directivité marquée.

\section{Conclusion}

Ce travail a abouti à la réalisation d'un logiciel de simulation numérique de champs acoustiques à partir de sources élémentaires (monopôles et dipôles). La définition de la position de ces sources dans l'espace et de leur phase relative permet de créer des situations d'interférence marquées et la définition d'une matrice de cohérence entre sources permet de constituer des structures de champs énergétiques propres aux sources 

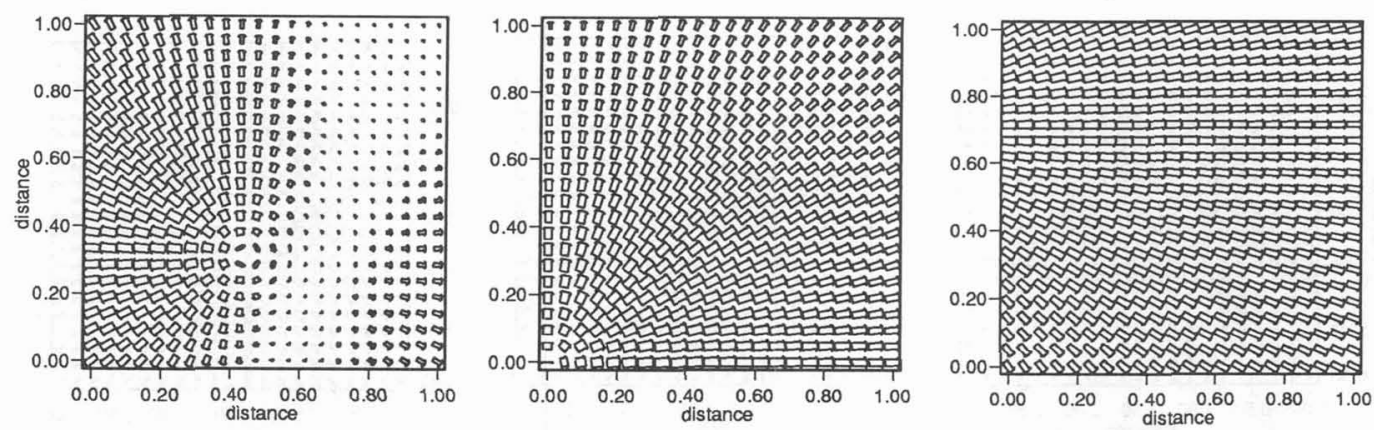

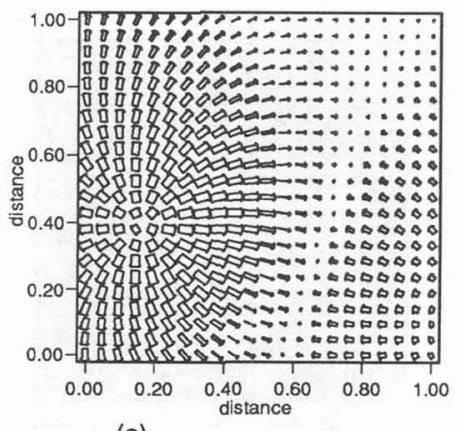

(a)

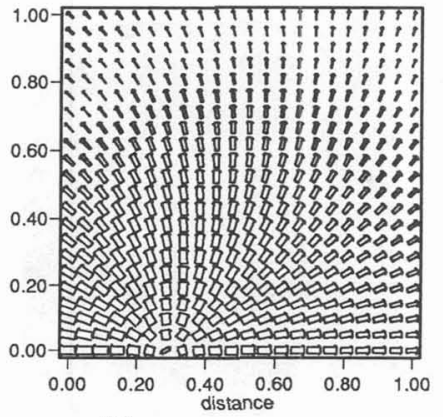

(b)

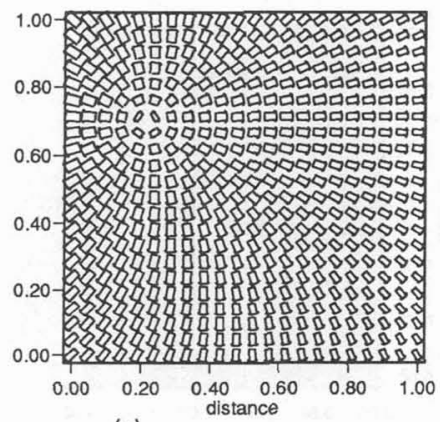

(c)

Figure 4 - Intensités acoustiques active (haut) et réactive (bas) d'un dipôle représentées sur différents plans d'observation $(F=500 \mathrm{~Hz})$; (a) Origine du plan $(0.01,-0.5,-0.5), \alpha_{1}=\pi / 6, \alpha_{2}=\pi / 6$; (b) Origine du plan $(0.5,0.5,0.01), \alpha_{1}=\pi / 4, \alpha_{2}=0$; (c) Origine du plan $(1,0.5,0.01), \alpha_{1}=0, \alpha_{2}=\pi / 4$.

larges bandes rencontrées dans les applications industrielles. Le choix de séparer le repère des sources et le repère de mesure ainsi que l'utilisation systématique de transformations de repères donne une grande souplesse pour orienter les surfaces d'observation autour des sources. Des exemples montrent combien la projection d'un champ de vecteurs (intensité active et réactive) créé par le rayonnement d'une source sur une surface de mesure située à distance peut être trompeuse si l'on veut utiliser cette information pour la localiser. L'emploi d'une méthode d'holographie acoustique pour effectuer une propagation inverse permet seule de lever le doute.

\section{Remerciements}

Ce travail a été réalisé avec le support de la Direction des Recherches, Etudes et Techniques (DRET).

\section{Références}

[1] F. Jacobsen, "Active and reactive, coherent and incoherent sound fields", J. Sound Vib. 130 (3), 493-507 (1989).

[2] G. Krishnappa, "Acoustic intensity in the nearfield of two interfering monopoles", J. Acoust. Soc. Am. 74 (4), 1291-1294 (1983).

[3] J.C Rebillat, S. Rifai, "Modèle plan du rayonnement acoustique de sources ponctuelles", J. Acoustique 3, (1990).

[4] T. Loyau, J.C. Pascal, P. Gaillard, "Broad-band acoustic holography reconstruction: BAHIM, an experimental method using acoustic intensity", Inter-Noise 88 Proc., Avignon, France, 30 Aug.- 1 Sept. 1988, 233-236.

[5] J.F. Li, J.C. Pascal, A. Garcia, C. Carles, "Simulation numérique", Rapport interne CETIM, Centre Technique des Industries Mécaniques, Senlis (1991). 